\title{
Perennial rhinitis
}

\author{
Hesham A Saleh, ${ }^{1}$ Stephen R Durham²
}

\begin{abstract}
${ }^{1}$ Charing Cross and Royal
Brompton Hospitals, London; and Imperial College of Medicine,

London

${ }^{2}$ Imperial College of Medicine,

National Heart and Lung Institute, London; and Royal Brompton

Hospital, London
\end{abstract}

Correspondence to: $\mathrm{H}$ Saleh

h.saleh@imperial.ac.uk

BMJ 2007;335:502-7

doi:10.1136/bmj.39304.678194.AE
Perennial rhinitis can be defined clinically as an inflammatory condition of the nose characterised by nasal obstruction, sneezing, itching, or rhinorrhoea, occurring for an hour or more on most days throughout the year. Rhinitis is commonly managed by both primary and secondary care physicians. Although most cases can be diagnosed and treated in primary care, referral to secondary care is often necessary when patients do not respond to treatment or other diagnoses are suspected.

A recent large scale, cross sectional study in six western European countries found that the overall prevalence of rhinitis was $23 \% .^{1}$ The study also showed that the condition is often undiagnosed, as $45 \%$ of patients with investigator confirmed allergic rhinitis had not previously received a diagnosis from their physicians. A published review of previous population based studies showed that, as with asthma, both seasonal and perennial rhinitis seem to be increasing. ${ }^{2}$

\section{How is perennial rhinitis classified? Allergic rhinitis}

Perennial allergic rhinitis can be more difficult to diagnose than seasonal allergy, particularly if the patient presents with secondary symptoms of sinusitis and a "permanent cold." The most common allergen to account for perennial allergy symptoms is the house dust mite (Dermatophagoides pteronyssinus). Other common causes are animals, particularly cats, dogs, and horses. In some parts of the world "typical" seasonal allergens are perennial in nature. Equally, symptoms of perennial rhinitis may not be present all year round.

\section{Box 1| Taking a history}

- Listen to patient's account of symptoms

- How long has the condition been present?

- Impact on lifestyle: how frequent and severe is it? Does it affect work, school, leisure time, sleep?

- Seasonal or perennial?

- Trigger factors: allergic or non-allergic?

- Exposure to allergens through occupation or hobbies?

- Allergens in the home

- Does patient have history of asthma, eczema, rhinitis?

- Drug or food induced?

- Family history

- Treatment: compliance, efficacy, side effects

-What is the main symptom?
For these reasons a new classification has been put forward in the document on allergic rhinitis and its impact on asthma (ARIA).$^{34}$ This subdivides allergic rhinitis in relation to the duration of symptoms into "intermittent" and "persistent." The severity of allergic rhinitis is also classified as "mild" or "moderate-severe" (fig 1). Recent cross sectional studies showed that a large number of patients classified by their doctor as having seasonal symptoms did in fact have persistent rhinitis, whereas many classified as having perennial rhinitis actually had intermittent rhinitis. Patients with persistent rhinitis had more severe symptoms and higher rate of self awareness and previous diagnosis of rhinitis; they were also clearly distinct in their sensitisation pattern and drug use $^{56}$ This supports the validity of the classification and is expected to simplify management decisions by allowing them to be tailored to the individual patient.

\section{Non-allergic rhinitis}

Patients with non-allergic rhinitis present with perennial or persistent symptoms, which can occasionally be attributed to certain factors; however, in a large number of patients no specific triggers can be found and the condition is termed "idiopathic rhinitis." To date, no precise data are available on the prevalence of nonallergic rhinitis, but it is considered to be less common than allergic rhinitis. Table 1 lists various types of nonallergic rhinitis with their causes and characteristics.

\section{What is the differential diagnosis?}

Structural abnormalities of the nose include deviation of the nose or septum, enlarged middle and inferior turbinates, adenoidal hypertrophy (particularly in children; rare in adults), and choanal atresia. Chronic rhinosinusitis is secondary to obstruction of the ostiomeatal complex, whether structural or secondary to an inflammatory condition. This is the area lying between the middle and inferior turbinates and the natural ostium of the maxillary sinus where the maxillary, anterior ethmoidal, and frontal sinuses drain. ${ }^{7}$

Nasal polyps result from inflammation of the mucosal lining of the sinuses ${ }^{8}$; the lining prolapses down, particularly from the anterior ethmoidal sinuses, through the middle meatus to obstruct the nasal airway (fig 2). Allergy does not seem to be an important factor. Nasal polyps in children are rare and are almost invariably associated with cystic fibrosis. In adults, a strong association exists between 


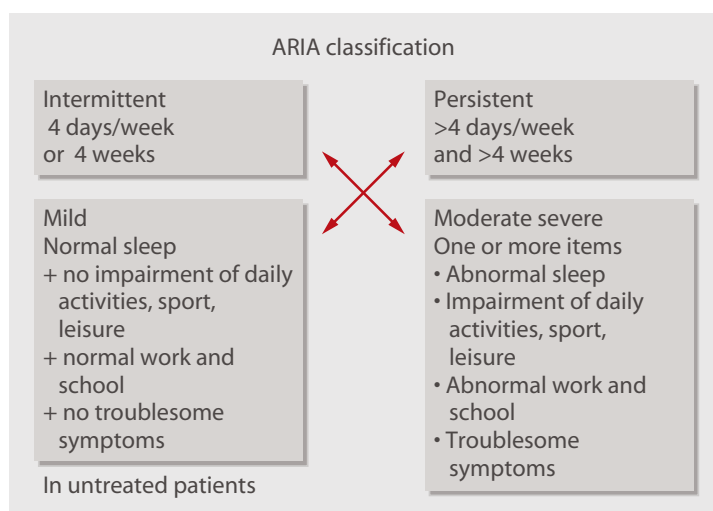

Fig 1 | Classification of allergic rhinitis. ${ }^{3}$ ARIA=allergic rhinitis and its impact on asthma

nasal polyps, asthma, and sensitivity to aspirin (Samter's triad). Granulomatous rhinitis may be associated with Wegener's granulomatosis and sarcoidosis (fig 3).

Leaking of cerebrospinal fluid will present with watery rhinorrhoea, often unilateral. It is usually associated with trauma (including surgical trauma) or neoplasia, but spontaneous leaking may occur. Nasal neoplasms are rare; the diagnosis should be considered in patients with unilateral symptoms of nasal obstruction, pain, or bleeding.

\section{How is the diagnosis made?}

The diagnosis is made on history and examination and is supported by skin prick testing. Taking a history need not be time consuming. A glance at the classification and differential diagnosis will suggest the most important questions (box 1). Rare, sinister causes of rhinitis need to be excluded. Unilateral symptoms should always be regarded with suspicion, particularly if associated with symptoms of increasing nasal obstruction, blood stained nasal discharge, or facial pain. ${ }^{9}$

Ear, nose, and throat surgeons examine the nose with a head mirror or headlight and a nasal speculum, supplemented by rigid or flexible nasendoscopy. In general practice, the nose can be examined with an auriscope fitted with the largest speculum. A large, swollen, oedematous inferior or middle turbinate can easily be confused with a polyp; polyps, however, unlike turbinates, are usually pale grey, translucent, and mobile and lack any sensation on gentle probing (fig 4).

\section{What investigations are needed? \\ Skin prick test}

Most cases of perennial allergic and non-allergic rhinitis need no specific investigations other than skin prick testing. Evidence from controlled trials shows the high sensitivity and specificity of skin tests. ${ }^{10}$ These provide supportive information for the history to diagnose specific allergies and, when negative, largely exclude IgE mediated disease. They are also important if avoidance measures are to be considered. When skin prick tests are not available or the patient is taking antihistamines or has dermatographism, total and allergen
Box 2 House dust mite avoidance measures

- Allergen proof bed sheets, duvets, and pillow cases should be used

- Sheets, blankets, and duvets should be washed at least once a week

- Furry toys or cushions should not be kept on beds; favourite toys should be washed regularly at temperatures of at least $60^{\circ} \mathrm{C}$

- Surfaces should be wiped with a clean, damp cloth

- If possible, wooden or other hard vinyl floorings should be chosen instead of carpets, and roller blinds that can be wiped clean should be fitted rather than curtains

- Woollen blankets or feather bedding should not be used in the home; synthetic pillows and acrylic duvets can be tried instead

specific IgE concentrations in the blood may be determined (radio-allergosorbent test-RAST-or enzyme linked immunosorbent assay_ELISA).

\section{Other tests}

If the history or examination suggests that other factors need to be excluded, the patient may need a variety of investigations, depending on the history and clinical findings. These are usually done in secondary care.

\section{Blood tests}

In chronic rhinosinusitis, full blood count and differential, including an eosinophil count, may be useful and immunoglobulin concentrations should be checked. If in doubt and infective rhinitis is suspected, specific IgG antibodies to tetanus and pneumococcus can be measured; if these are low, the measurements can be repeated six weeks after specific vaccination with tetanus or pneumovax.

\section{Erythrocyte sedimentation rate}

Erythrocyte sedimentation rate is indicated if rhinitis as a presenting feature of vasculitis such as Churg-Strauss syndrome is suspected. Blood tests for antineutrophil cytoplasmic antibody or angiotensin converting enzyme may be indicated if Wegener's granulomatosis or nasal sarcoidosis is suspected. It is also important to consider whether the patient may be HIV positive or be

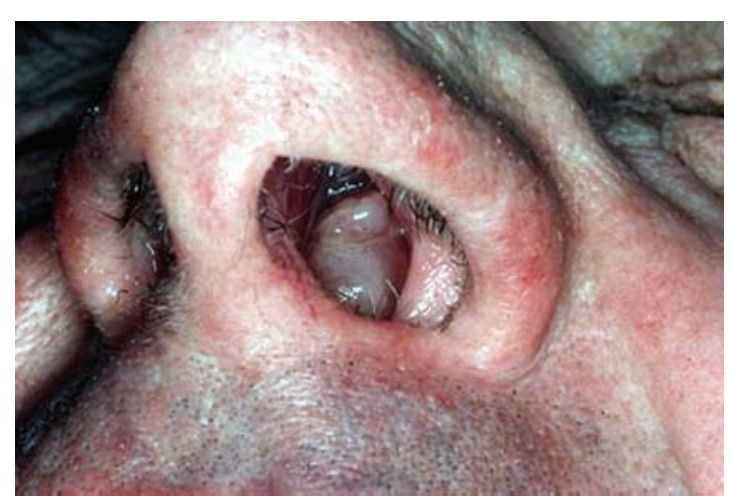

Fig 2 | Nasal polyps 


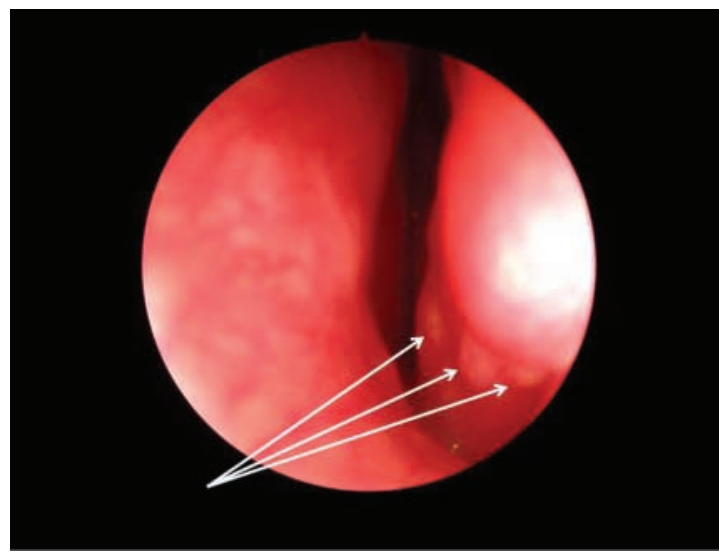

Fig 3 | Endoscopic view of nasal sarcoidosis, showing granulomas on left inferior turbinate (arrows)

compromised by treatment with immunosuppressant drugs that predispose to infective rhinitis.

\section{Imaging}

Computed tomography scanning of the sinuses is indicated when medical treatment has failed, the diagnosis of chronic rhinosinusitis is suspected and could not be confirmed on history and examination, or neoplasia is suspected. Evidence shows that plain radiographs of the sinuses can be misleading, and computed tomography of the sinuses in the coronal plane has become the standard international imaging method. ${ }^{11}$ Good quality cross sectional studies show that some mucosal thickening is often present on computed tomography scans of patients with allergic rhinitis, but the diagnosis of chronic rhinosinusitis is asserted when obstruction of the ostiomeatal complex is present. ${ }^{12}$

\section{Further tests}

Further tests are considered to exclude rarer conditions and are usually done in highly specialised centres, where some of them are mainly used for
Box 3 Surgical treatment (evidence levels 3 and 4)

- The first line of treatment for allergic or non-allergic perennial rhinitis is medical

- When drugs fail and a structural abnormality exists, surgery may be indicated

- Surgical reduction of the inferior turbinates or correction of a deviated nasal septum or nose may be needed to improve the airway or at least to improve access for topical medical treatment

- Surgery continues to have a major role in the management of nasal polyps and sinusitis when these conditions fail to respond to medical treatment

- The management of nasal polyps and sinusitis has improved with the introduction of minimally invasive endoscopic sinus surgery

research purposes. Nasal inspiratory peak flow can be measured with a modified peak flow meter. This test is easy and inexpensive to do, but forced inspiration may be associated with vestibular collapse. Rhinomanometry records resistance in the nasal airway by measuring nasal airflow with a face mask and pneumotachograph, and pressure gradient from the front to the back of the nose with a manometer. Acoustic rhinometry measures the cross sectional area of the nasal cavity and is much easier to do.

Nasal allergen challenge is occasionally indicated when a strong history exists in the face of negative skin prick test or radio-allergosorbent test. Patients with suspected occupational rhinitis due to a sensitiser in the work place may need an occupational type provocation in which simulated exposure to the suspected agent is reproduced in an isolation cubicle. Assessment of nasal mucociliary clearance is indicated when ciliary dyskinesia is suspected. This is assessed simply by measuring the time taken for the patient to detect a sweet taste after a $0.5 \mathrm{~mm}$ particle of saccharin is placed on the mucosa of the inferior turbinate. If the test result is abnormal, further

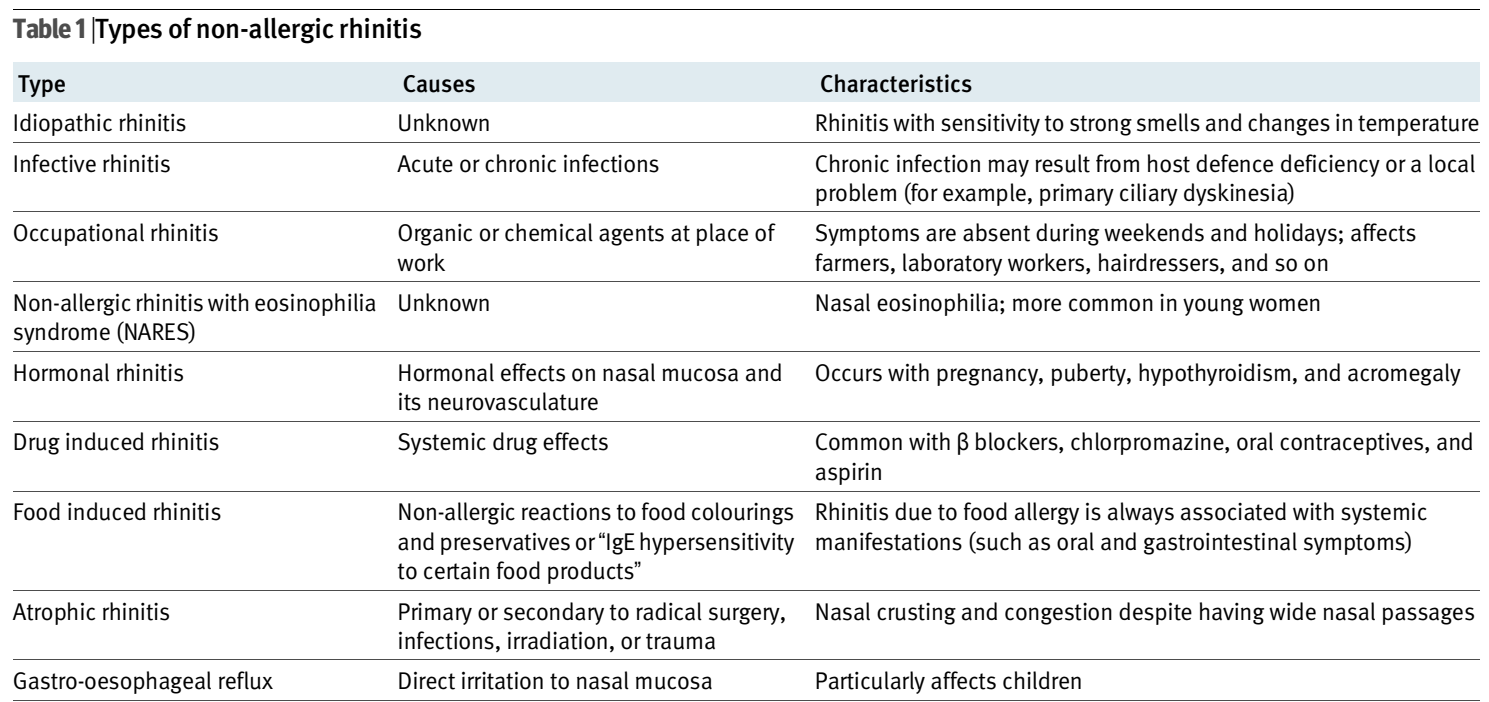



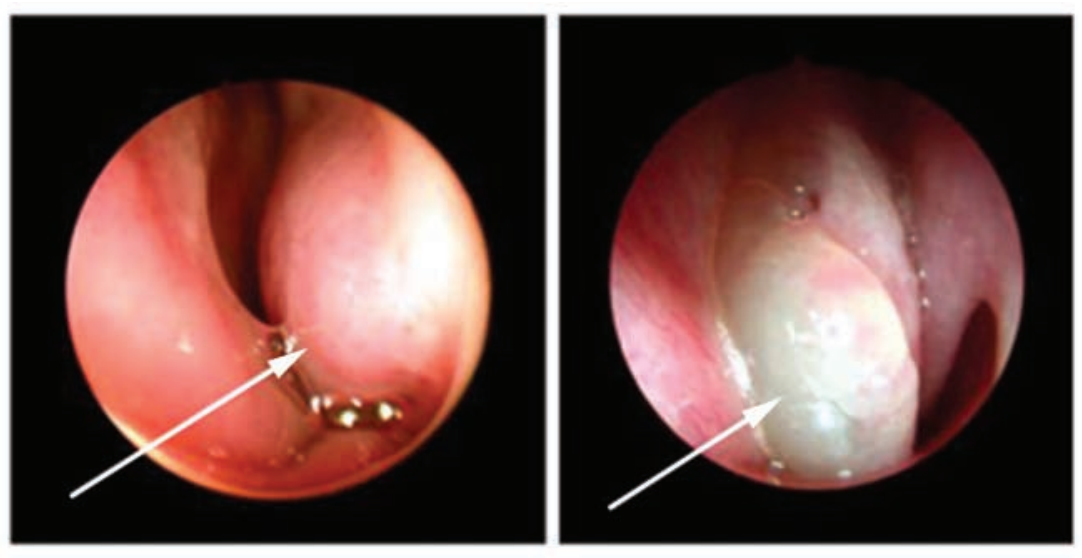

Fig 4 | Endoscopic view of enlarged left inferior turbinate (arrow) in patient with perennial rhinitis (left), compared with patient with characteristic nasal polyps (arrow) (right)

assessment of ciliary function is done in specialist rhinology clinics and involves taking a brushing of the nasal mucosa overlying the inferior turbinate and measuring the frequency of the beating cilia detected with a microscope attached to a photometric cell (normal range 12-15 Hz). Exhaled nasal nitric oxide is a sensitive marker of inflammation and can be measured through a face mask. This is currently used primarily in research trials but is diagnostic of primary ciliary dyskinesia in patients without severe nasal obstruction.

\section{Swabs, smears, and biopsies}

With infective symptoms, swabs for culture and sensitivity may be useful; nasal smears for cytology may show high concentrations of eosinophils; and biopsies for histology may be indicated when investigating granulomatous conditions or neoplastic disease. Olfactory thresholds can be assessed by the readily available "scratch and sniff" tests that use cards impregnated with microencapsulated odorants.

\section{How is perennial rhinitis treated?}

A stepwise approach according to the severity of symptoms, based on the available randomised trials, has been adopted by ARIA (fig 5). ${ }^{4}$ Main lines of treatment are allergy avoidance, antihistamines, and topical

Table 2 |Comparative effects of various agents on rhinitis associated symptoms (from consensus statement) $^{17}$

\begin{tabular}{lccccc} 
& Sneezing & Rhinorrhoea & $\begin{array}{c}\text { Nasal } \\
\text { obstruction }\end{array}$ & Nasal itch & Eye symptoms \\
$\mathrm{H}_{1}$ antihistamines: & & ++ & + & +++ & ++ \\
\hline Oral & ++ & ++ & + & ++ & 0 \\
\hline Intranasal & ++ & 0 & 0 & 0 & +++ \\
\hline Intraocular & 0 & +++ & +++ & ++ \\
\hline Corticosteroids & +++ & + & & + & 0 \\
\hline Cromoglycates: & & + & + & 0 & ++ \\
\hline Intranasal & + & 0 & 0 & 0 & 0 \\
\hline Intraocular & 0 & ++ & ++ & 0 & ++
\end{tabular}

steroids. Patients with non-allergic rhinitis are more difficult to treat, but many respond to topical steroids. If a particular factor for non-allergic rhinitis has been identified (such as a drug), symptoms can usually be eliminated by its exclusion. Patients may need long term or permanent treatment. Long term prognosis is not well documented, but patients with perennial rhinitis are generally thought to improve over time. ${ }^{4}$

\section{Allergen avoidance}

Perennial allergic rhinitis is commonly associated with allergy to house dust mite. The routine implementation of mite avoidance measures has been questioned in a recent Cochrane systematic review. ${ }^{13}$ However, whether effective reduction of mite levels was achieved in many studies was not clear. Two studies in adultsone in perennial rhinitis and one in bronchial asthmashowed that a single intervention with mite proof bed covers was unsuccessful, and clearly such measures should not be recommended in isolation. ${ }^{14}$ Multiple avoidance measures are still thought to be effective and should in our opinion be recommended (box 2).

Patients who are found to be allergic to furred animals should reduce their exposure as much as possible and be discouraged from having pets in the home, although psychosocial consequences often have to be considered. Occupational allergy requires prompt recognition and avoidance of the offending agent if long term consequences are to be avoided.

\section{Medical treatment}

Table 2 shows the comparative effectiveness of drugs for allergic rhinitis. ${ }^{4-15}$ A recent review of randomised controlled trials confirmed the efficacy of antihistamines in persistent allergic rhinitis. ${ }^{16}$ Most patients will attain good control on intranasal steroids, and a large body of data shows that they are effective for all symptoms of allergic perennial rhinitis, including nasal obstruction, itching, sneezing, and watery rhinorrhoea. ${ }^{4}$ Modern intranasal steroids are safe for long term use in adults when used within the recommended dosage. In children, they should be used at the lowest dose that controls symptoms, particularly when used concurrently with other inhaled or intranasal steroids. ${ }^{17}$ Occasionally, intranasal steroids may be associated with dryness, crusting, or slight bleeding, which if recurrent may necessitate withdrawal of treatment.

The chromone sodium cromoglycate is less effective than antihistamines and corticosteroids and needs

\section{SOURCES AND SELECTION CRITERIA}

We searched Medline by using the keywords "rhinitis", "perennial rhinitis", "persistent rhinitis", "diagnosis", and "management". We also searched the Cochrane Database of Systematic Reviews with the keywords "rhinitis", "perennial rhinitis", and "persistent rhinitis". We also consulted personal archives and documents on the subject 


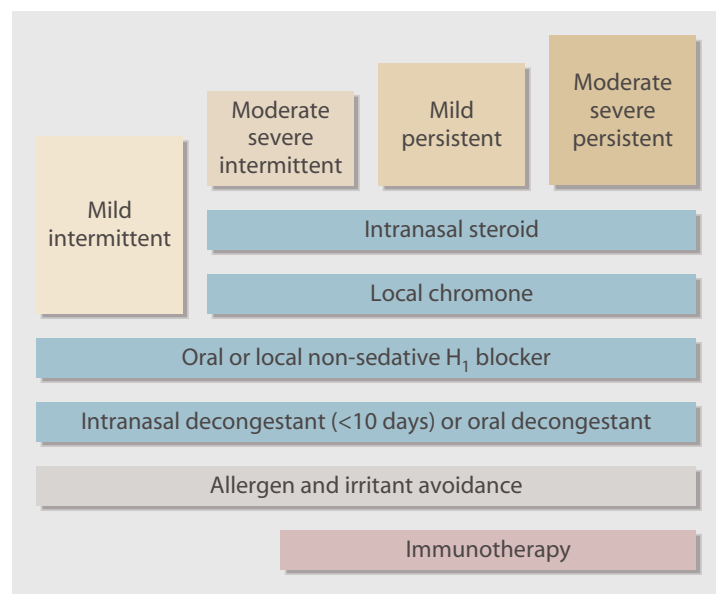

Fig 5 | Stepwise approach to treatment of perennial rhinitis ${ }^{4}$

frequent use (up to five times daily), which may compromise compliance. The anticholinergic intranasal agent ipratropium bromide is effective in controlling watery rhinorrhoea, particularly if this is the dominant symptom. The dose may need to be titrated against symptoms. Oral antileukotrienes have been shown to be effective in allergic rhinitis in randomised controlled trials. ${ }^{18}$ Antileukotrienes may be beneficial in patients with aspirin sensitivity.

Other treatments such as nasal decongestants and oral steroids should be used only in certain situations. Topical nasal decongestants may be useful at the start of treatment to "open up" the nose and should be used for less than two weeks to avoid the risk of developing "rhinitis medicamentosa," in which patients develop rebound congestion. Oral steroids should be used only as a last resort when other treatments have failed. Oral steroids are extremely effective and can be used in

\section{TIPS FOR NON-SPECIALISTS}

\section{What to exclude (differential diagnosis)}

- Structural abnormalities that cause nasal obstruction such as deviation of the septum, enlarged middle and inferior turbinates, adenoidal hypertrophy (particularly in children; rare in adults), and choanal atresia

- Chronic rhinosinusitis, in which patients may have purulent anterior or posterior discharge, sinus pressure or pain, and nasal obstruction or congestion

- Nasal polyps, which can be characterised by severe nasal obstruction, loss of sense of smell, and greyish insensitive swellings on nasal examination

- Rarely, granulomatous rhinitis may be associated with Wegener's granulomatosis and sarcoidosis

- Leaking of cerebrospinal fluid, either secondary to trauma or spontaneous, will present with watery rhinorrhoea, often unilateral

When to refer to a specialist

- If the diagnosis is in doubt

- If medical treatment of rhinitis was not successful

- If the patient has unilateral symptoms of nasal obstruction, pain, or bleeding

- If the patient has a suspected structural abnormality, nasal polyps, granulomas, or cerebrospinal fluid leak-in the case of chronic rhinosinusitis, medical treatment should be tried first

\section{ADDITIONAL EDUCATIONAL RESOURCES}

\section{Resources for health professionals}

Durham SR, ed. $A B C$ of allergies. London: BMJ

Publishing Group, 1998

Dykewicz MS, Fineman S, eds. Diagnosis and management of rhinitis: complete guidelines of the Joint Task Force on Practice Parameters on Allergy, Asthma and Immunology. www.jcaai.org/Param/ Rhinitis/a011x8004780.pdf

The Cochrane collaboration. Ear, nose and throat disorders. www.cochrane.org/reviews/en/topics/60. html

British Society for Allergy \& Clinical Immunology (www. bsaci.org) -A valuable educational site with references to various courses and conferences

\section{Resources for patients}

Patient UK (www.patient.co.uk)—Covers various aspects of rhinitis, including diagnosis and management, with references to various relate websites and support groups

BBC Health (www.bbc.co.uk/health/conditions/ allergies)—Good information for patients, with questions and answers section

severe allergic rhinitis and nasal polyps. ${ }^{4-15}$ Short courses of an oral steroid should be used rather than depot injections. They should be avoided in patients with known contraindications, in children, and in pregnant women.

\section{Surgery}

Surgical treatment is indicated only in patients with specific nasal abnormalities (box 3). Many cohort studies (prospective case studies) support the efficacy of surgery in treating structural abnormalities, ${ }^{1920}$ but randomised trials are rare because a placebo is not possible.

\section{Immunotherapy}

Allergen immunotherapy by the subcutaneous route and, more recently, by the sublingual route has been shown to be effective in both seasonal and perennial allergic rhinitis in patients with proved $\mathrm{IgE}$ mediated disease and a limited spectrum of allergies. ${ }^{21}$ Sublingual treatment is currently available only for grass pollen allergy, but specific agents for perennial allergens are being trialled. Subcutaneous immunotherapy in children with mite allergy has also been shown to reduce the onset of new allergic sensitisations. In perennial rhinitis, its use is confined to those patients in whom a perennial allergen is the dominant cause and in whom avoidance measures and medical treatment are either not effective or not tolerated owing to side effects. The presence of chronic bronchial asthma is a contraindication in the United Kingdom. The treatment should be prescribed only by trained and experienced physicians, and the subcutaneous form should be administered in a specialist clinic with access to resuscitation equipment in view of the risk of systemic side effects. ${ }^{22}$ 


\section{SUMMARY POINTS}

Perennial allergic rhinitis is a common condition in general practice

The most common allergen is the house dust mite, followed by cats and dogs

Diagnosis is through history and skin prick testing

Patients with unilateral symptoms, especially if they have pain or bleeding, should be referred to an ear, nose, and throat specialist

Avoidance measures should be taken where appropriate

Medical treatment, mainly with antihistamines, topical

corticosteroids, or both, is usually highly effective

Immunotherapy is reserved for severe cases in which avoidance measures and medical treatment are either not effective or not tolerated

Surgery is reserved for certain patients who have structural abnormalities

Contributors: HAS collected the data and wrote the first draft. SRD revised and added to the manuscript and provided further references. HAS is the guarantor.

Competing interests: None declared.

Provenance and peer review: Commissioned; externally peer reviewed.

1 Bauchau V, Durham SR. Prevalence and rate of diagnosis of allergic rhinitis in Europe. Eur Respir J 2004;24:758-64.

2 Sly RM. Changing prevalence of allergic rhinitis and asthma. Ann Allergy Asthma Immunol 1999;82:233-48.

3 Bousquet J, Van Cauwenberge P, Khaltaev N. Allergic rhinitis and its impact on asthma. J Allergy Clin Immunol 2001;108:S147-334.

4 Bousquet J, van Cauwenberge P, Aït Khaled N, Bachert C, Baena-Caqnani CE, Bouchard J, et al. Pharmacologic and anti-lgE treatment of allergic rhinitis ARIA update (in collaboration with GA2LEN). Allergy 2006;61:1086-96.

5 Bauchau V, Durham SR. Epidemiological characterization of the intermittent and persistent types of allergic rhinitis. Allergy 2005;60:350-3.

6 Bousquet PJ, Bousquet-Rouanet L, Co Minh HB, Urbinelli R, Allaert FA, Demloy P. ARIA (allergic rhinitis and its impact on asthma) classification of allergic rhinitis severity in clinical practice in France. Int Arch Allergy Immunol 2007;143:163-9.
7 Messerklinger W. Role of the lateral nasal wall in the pathogenesis, diagnosis and therapy of recurrent and chronic rhinosinusitis. Laryngol Rhinol Otol 1987;66:293-9.

8 Saleh HA, Lund VJ. Treating nasal polyps. Practitioner 2000;244 (1607):84-6, 88-9, 91-3.

9 Brookes N, Saleh H, Mackay I. 10-minute consultation: rhinitis. Referral to specialist otolaryngologist may be advisable. BMJ 2002;324:1219.

10 Carrr WW. Improvements in skin-testing technique. Allergy Asthma Proc 2006;27:100-3.

11 Zinreich SJ. Paranasal sinus imaging. Otolaryngol Head Neck Surg 1990;103:863-8.

12 Krouse JH. Computed tomography stage, allergy testing, and quality of life in patients with sinusitis. Otolaryngol Head Neck Surg 2000;123:389-92.

13 Sheikh A, Hurwitz B, Shehata Y. House dust mite avoidance measures for perennial allergic rhinitis. Cochrane Database Syst Rev 2007;(1):CD001563.

14 Terreehorst I, Hak E, Oosting AJ, Tempels-Pavlica Z, de Monchy JGR, Bruijnzeel-Koomen C, et al. Evaluation of impermeable covers for bedding in patients with allergic rhinitis. $N$ Engl J Med 2003;349:237-46

15 Van Cauwenberge P, Bachert C, Passalacqua G, Bousquet J, Canonica GW, Durham SR, et al. Consensus statement on the treatment of allergic rhinitis. Allergy 2000;55:116-34.

16 Hore I, Georgalas C, Scadding G. Oral antihistamines for the symptom of nasal obstruction in persistent allergic rhinitis-a systematic review of randomized controlled trials. Clin Exp Allergy 2005;35:207-12.

17 Al Sayyad J, Fedorowicz Z, Alhashimi D, Jamal A. Topical nasal steroids for intermittent and persistent allergic rhinitis in children. Cochrane Database Syst Rev 2007;(1):CD003163.

18 Wilson AM, O'Byrne PM, Parameswaran K. Leukotriene receptor antagonists for allergic rhinitis: a systematic review and metaanalysis. Am J Med 2004;116:338-44.

19 Stewart MG, Smith TL, Weaver EM, Witsell DL, Yueh B, Hannley MT, et al. Outcomes after nasal septoplasty: results from the nasal obstruction septoplasty effectiveness (NOSE) study. Otolaryngol Head Neck Surg 2004;130:283-90.

20 Hopkins C, Browne JP, Slack R, Lund V, Topham J, Reeves B, et al. The national comparative audit of surgery for nasal polyposis and chronic rhinosinusitis. Clin Otolaryngol 2006;31:390-8.

21 Wilson DR, Lima MT, Durham SR. Sublingual immunotherapy for allergic rhinitis: systematic review and meta-analysis. Allergy 2005;60:4-12.

22 Alvarez Cuesta E, Bousquet J, Canonica WG, Durham SR, Malling H, Valovirta E. Standards for practical immunotherapy. Allergy 2006;61(suppl 82):1-20.

Accepted: 1 August 2007

\section{Life class lives again}

The teachers at Homerton School of Art were worried that their students could draw still life, but not movement. They made a film with five nude models, always on the move, while the students made drawings on paper arranged on the floor. As a visiting lecturer at Bradford College of Art, I was invited to see the film, and my thoughts turned to my own students - making one or two drawings in a two hour practical and completing them from a textbook at home.

Next practical I gave my students paper, pencils, and a board to draw on - and released 13 frogs from a box. The rules were simple: each student had to choose a frog and follow it, making as many drawings as possible in the next 20 minutes, and then return the frog to me. It was fun and noisy, frogs and students crawled under and over the benches. Colleagues, attracted by the noise looked in from the corridor. One student made 19 drawings in 23 minutes. Seven showed marked improvement. The students had the last laughwatching me trying to put 13 very active frogs back in their small box, with frogs jumping out again as I put in the last ones. I published a short paper showing the improvements in students' drawing: "The running, jumping and standing still game.'

Next year we visited Moscow for a microbiological conference, taking a translation of his book to Kornei Chukovsky, the great Russian man of letters. Only on the last afternoon did we manage to reach his house in the writers' village. Sitting with him on a tree trunk in his children's garden theatre, my wife told him of my frogs. Turning to me, he said, "They don't let you teach that anymore, do they?"

"No," I said, "but how did you know ?"

"It's the same everywhere," he replied.

Several years later, at dinner at a conference on teaching, I remarked that in science one received requests for reprints and other scientists cited one's work, but no one had ever written to me for a paper about teaching. "Did anyone ever read what I had written?" I asked, citing my frog paper.

Someone further down the table answered, "Yes, that paper changed my life."

H V Wyatt visiting lecturer in philosophy, University of Leeds, Leeds nurhvw@leeds.ac.uk 